transplantation
of human islets
is an effective
treatment
for T1DM
complicated
by impaired
awareness of
hypoglycemia
and severe
hypoglycemic
episodes

$\Rightarrow$ DIABETES

\title{
Islet transplantation for T1DM
}

Transplantation of human pancreatic islets could prevent life-threatening hypoglycaemic events in patients with type 1 diabetes mellitus (T1DM) who have not responded to other therapies, according to a new clinical trial.

In patients with T1DM, ineffective insulin-mediated glycaemic control can result in hypoglycaemia. Recurring low blood glucose levels can impair awareness of hypoglycaemia, which might lead to severe hypoglycaemic events that could produce fatal outcomes such as seizures and coma. Preliminary studies have suggested that transplantation of human pancreatic islets might be effective in individuals for whom other interventions have been unsuccessful, but this procedure is currently considered experimental and its efficacy is unproven.
A team of investigators, therefore, carried out a multicentre, single arm phase III trial of 48 adults with T1DM who had persisting impaired awareness of hypoglycaemia and severe hypoglycaemic events, despite receiving conventional treatment. Each participant underwent immunosuppression and one or more transplants of purified human pancreatic islets. The primary trial end point was met in $87.5 \%$ of the participants, who achieved an $\mathrm{HbA}_{1 \mathrm{c}}$ of $<7 \% 1$ year after islet transplantation, with no severe hypoglycaemic events 28-365 days after the procedure. "This trial demonstrates that transplantation of human islets is an effective treatment for [T1DM] complicated by impaired awareness of hypoglycaemia and severe hypoglycaemic episodes," remarks lead author Bernhard Hering.

\section{A minority of patients} experienced adverse effects such as infections or kidney dysfunction as a consequence of the immunosuppression required for the transplant. Nevertheless, the researchers recommend that islet transplantation should be considered to protect patients with T1DM for whom other therapies have failed. A follow-up study is now underway to assess the long-term effects of islet implantation in these patients, which could provide further evidence for the potential licensure of this procedure.

Charlotte Ridler

ORIGINAL ARTICLE Hering, B. J. et al. Phase 3 trial of transplantation of human islets in type 1 diabetes complicated by severe hypoglycemia. Diabetes Care http://dx.doi.org/10.2337/ dc15-1988 (2016) 